\title{
Análisis Multivariado Aplicado a la Etapa de Deshidratación en la Técnica de Plastinación del Riñón de Caballo
}

\author{
Multivaried Analysis Applied at the Stage of Dehydration \\ in the Plastination Technique in Horse's Kidney
}

"Rivera, M. C.; "**Bonino, F.; "Fioretti, C.; "Galán, M.; "Gigena, S.; "Moine, R.; "Mouguelar, H.; "Natali, J. \& ** Quinteros, R.

RIVERA, M. C.; BONINO, F.; FIORETTI, C.; GALÁN, M.; GIGENA, S.; MOINE, R.; MOUGUELAR, H.; NATALI, J. \& QUINTEROS, R. Análisis multivariado aplicado a la etapa de deshidratación en la técnica de plastinación del riñón de caballo. Int. J. Morphol., 27(3):855-859, 2009.

RESUMEN: La Plastinación es la técnica más moderna para la conservación de piezas anatómicas y especímenes. Esta técnica consta de cuatro etapas: fijación, deshidratación, impregnación forzada y curado. Este trabajo pretende mejorar la eficiencia en la producción de órganos plastinados mediante la técnica de Plastinación estándar denominada S 10. Se utilizaron riñones de equinos mestizos criollos provenientes del Frigorífico Aimar S/A, ubicado en la zona rural de Río Cuarto. Luego de realizar la fijación del órgano con formalina se procedió a deshidratarlo. La deshidratación se llevó a cabo en tres sub-etapas donde en cada una de ellas se trabajó a la misma temperatura y se utilizó como solvente una solución de acetona en diferentes concentraciones. Las mediciones se realizaron con acetómetro registrándose el tiempo insumido para lograr la deshidratación. Los datos se analizaron estadísticamente por medio de: análisis de la varianza multivariada y análisis de correlación simple utilizando el paquete estadístico InfoStat. Los resultados muestran que los tiempos de deshidratación se ven influenciados por la edad del animal pero no por la posición ni por el peso del órgano.

PALABRAS CLAVE: Plastinación; Acetona; Deshidratación; Riñones; Caballo.

\section{INTRODUCCIÓN}

La plastinación es una auténtica revolución en el campo de la conservación de órganos reales. Gracias a esta técnica es posible ver, tocar y conservar órganos, piezas anatómicas y especímenes. En docencia, mejora la calidad de la enseñanza práctica y en investigación avanza en el campo de la anatomía topográfica como base para la interpretación de técnicas modernas de diagnóstico por imagen como resonancia magnética, endoscopía, tomografía computarizada, etc.

Las actuales técnicas de conservación a base de formol que se utilizan en el área de anatomía de muchas universidades no son las más adecuadas. Estas presentan desventajas, como por ejemplo, la rigidez de las piezas formuladas y la pérdida del color natural. Por otro lado, las propiedades físico-químicas del formol lo convierten en un gas, el cuál afecta a las personas que lo manipulan, irritando su mucosa ocular. Su inhalación produce irritación a la mucosa nasal y al tracto respiratorio superior e incluso puede afectar los pulmones. En tiempo de exposición prolongada irrita los tejidos cutáneos (Ballenguer, 1984; Olsen et al., 1984).

La técnica, desarrollada por Gunther von Hagens en Heidelberg, Alemania, se fundamenta principalmente en la sustitución de los líquidos tisulares (agua y lípidos) por polímeros (silicona o resina epóxica) y consta básicamente de cuatro etapas: fijación: luego de realizada la disección el especimen u órgano se fija en una solución de formol al 5\% por un período de una semana; deshidratación, este proceso se realiza con un solvente orgánico (acetona) y tiene por finalidad la extracción del agua tisular; impregnación forzada: aquí el solvente es reemplazado por un polímero en una cámara de vacío a bajas temperaturas; curado: en esta etapa

\footnotetext{
* Anatomía Animal, Depto. de Anatomía Animal. Facultad de Agronomía y Veterinaria. Universidad Nacional de Río Cuarto, Argentina.

** Matemáticas y Bioestadística. Facultad de Agronomía y Veterinaria. Universidad Nacional de Río Cuarto, Argentina.

Este trabajo fue subsidiado por SECYT-UNRC (18/A228).
} 
RIVERA, M. C.; BONINO, F.; FIORETTI, C.; GALÁN, M.; GIGENA, S.; MOINE, R.; MOUGUELAR, H.; NATALI, J. \& QUINTEROS, R. Análisis multivariado aplicado a la etapa de deshidratación en la técnica de plastinación del riñón de caballo. Int. J. Morphol., 27(3):855-859, 2009.

se lleva a cabo el endurecimiento del polímero por medio del empleo de un catalizador (von Hagens et al., 1987). Los especímenes plastinados están libres de olor, tienen alta durabilidad, son secos y muy resistentes. En la Universidad Nacional de Río Cuarto se ha comenzado a implementar la técnica trabajando en las dos primeras etapas de la misma utilizando ambos riñones de equinos jóvenes y adultos procedentes del Frigorífico Aimar S/A ubicado en la región de Río Cuarto, Argentina.

El objetivo del trabajo fue analizar como influye la edad, lateralidad del órgano (derecho o izquierdo) y el peso del riñón de caballo sobre el tiempo de deshidratación en la técnica de Plastinación.

\section{MATERIAL Y MÉTODO}

El presente trabajo se ha realizado en el Departamento de Anatomía de la Facultad de Agronomía y Veterinaria de la Universidad Nacional de Río Cuarto, Argentina. Se han utilizado 10 riñones derechos y 10 riñones izquierdos de caballo mestizos criollos provenientes del Frigorífico Aimar S/A ubicado en la zona rural de Río Cuarto. De los 10 pares de riñones, 5 pertenecían a animales jóvenes y los otros 5 a animales adultos, la edad se determinó por dentición. Luego de ser aislados de la cavidad abdominal mediante disección convencional, los riñones se fijaron con formalina al 5\% a $5^{\circ} \mathrm{C}$, por un período de 7 días. A cada riñón se le tomó el peso inicial antes de proceder a la deshidratación.

A fin de evitar roturas de los tejidos, la deshidratación del órgano se realizó en forma gradual. El proceso de deshidratación se dividió en tres sub-etapas (TD1; 2 y 3) consecutivas utilizando una solución de acetona-agua en concentraciones crecientes: $85 \%$ v/v (sub-etapa 1), $90 \%$ v/v (subetapa 2) y $99 \% \mathrm{v} / \mathrm{v}$ (sub-etapa 3). La temperatura durante el procedimiento se mantuvo en $-18^{\circ} \mathrm{C}$.

Durante cada sub-etapa se hizo el monitoreo periódico de la evolución en la concentración de la solución de lavado. Cuando la concentración de acetona en la solución disminuye en un $10 \%$ se da por finalizada la sub-etapa y se pasa a la siguiente. Se utilizó para estas mediciones un acetómetro calibrado para trabajar a $5^{\circ} \mathrm{C}$. Los datos obtenidos, concentración de la solución y tiempo insumido se registraron en planillas.

Análisis estadísticos para las variables edad y lateralidad (derecho o izquierdo) del riñón: se utilizó la prueba de Varianza Multivariada, considerando un modelo de efectos fijos de dos vías con interacción.
Modelo estadístico teórico:

$$
\begin{gathered}
\mathrm{Y} l k r=\mathrm{m}+\mathrm{t} l+\mathrm{b} k+\mathrm{g} l k+\mathrm{e} l k r \\
1=1 ; \ldots ; \mathrm{g} \\
\mathrm{k}=1 ; \ldots ; \mathrm{b} \\
\mathrm{r}=1 ; \ldots ; \mathrm{n}
\end{gathered}
$$

La variable respuesta es el tiempo de deshidratación, variable $\mathrm{p}$ dimensional que da origen a un vector respuesta de $p$ componentes. Los factores en estudio son la edad del animal y lateralidad del órgano (derecho o izquierdo).

\section{El modelo planteado para nuestro caso particular es:}

$$
\begin{aligned}
\mathrm{TD} l k r=\mathrm{m}+ & E_{1}+L_{\mathrm{k}}+(E L)_{\mathrm{lk}}+\mathrm{e}_{\mathrm{lkr}} \\
l & =1 ; \ldots ; 2 \\
k & =1 ; \ldots ; 2 \\
r & =1 ; \ldots ; 5
\end{aligned}
$$

Donde:

$T D$ es el vector respuesta, conteniendo los datos del tiempo de deshidratación, con $\mathrm{p}=3$ dimensiones y $r=5$ repeticiones (cinco mediciones del tiempo de deshidratación para cada una de las tres subetapas).

$E l$ es el factor edad, con $l=2$ niveles, jóvenes y adultos.

$L k$ es el factor de lateralidad del órgano, con $k=2$ niveles, derecho e izquierdo.

$(E L)_{\mathrm{lk}}$ corresponde a la interacción entre los dos factores.

$\mathrm{e}_{\mathrm{kr}}$ es el término del error aleatorio.

Tomando como referencia el modelo planteado para este trabajo, lo primero que se estudia es la posible interacción entre los factores (edad y lateralidad del órgano), mediante el contraste de las siguientes hipótesis:

$H_{0}: E L_{11}=E L_{12}=\ldots=E L_{1 \mathrm{k}}=0 \quad H_{1}:$ Al menos una $E L_{l k} \neq 0$

Si se rechaza la hipótesis nula se pueden estudiar por separado el efecto de cada uno de los factores mediante las siguientes hipótesis:

Influencia por factor Edad:

$$
H_{0}: E_{1}=E_{2}=0 \quad H_{1}: \text { Al menos un } E_{1} \neq 0
$$

Influencia por factor lateralidad del órgano:

$$
H_{0}: L_{1}=L_{2}=0 \quad H_{1}: \text { Al menos un } L_{k} \neq 0
$$

Análisis estadísticos para la variable peso del riñón. Se utilizó la prueba de correlación lineal simple. Las dos variables aleatorias con las que se trabajan fueron: tiempo de deshidratación en cada sub-etapa y el peso del riñón. Realizando las pruebas de hipótesis apropiadas se pueden obtener resultados que permitan arribar a una conclusión a fin de alcanzar el objetivo propuesto. 
RIVERA, M. C.; BONINO, F.; FIORETTI, C.; GALÁN, M.; GIGENA, S.; MOINE, R.; MOUGUELAR, H.; NATALI, J. \& QUINTEROS, R. Análisis multivariado aplicado a la etapa de deshidratación en la técnica de plastinación del riñón de caballo. Int. J. Morphol., 27(3):855-859, 2009.

\section{RESULTADOS}

La Tabla I muestra que los valores medios del TD en la sub-etapa 1 son notablemente menores que en la sub-etapa 2 y sub-etapa 3. También se observa en dicha tabla que los valores del tiempo de deshidratación en la sub-etapa 1 presentan más homogeneidad. Los valores medios de las tres sub-etapas del TD discriminados por edad y lateralidad del órgano se muestran en Tabla II.

Tras aplicar la matriz de covarianza a los valores obtenidos en cada sub-etapa del TD se obtiene los resulta- dos señalados en la Tabla III. En ella se muestra la variabilidad de cada variable y la covarianza entre cada variable.

La Tabla IV muestra todos los procedimientos de Análisis de la Varianza Multivariado (MANOVA) desarrollados. En la Tabla V se presenta el análisis de la varianza aplicado a los factores edad, lateralidad del órgano e interacción entre ambos factores. En las figuras 1, 2 y 3 se observa que las nubes de puntos no muestran tendencias como para marcar una posible ausencia de correlación entre las variables TD y peso del órgano. El coeficiente de correlación lineal para la variable peso del órgano en cada subetapa en el TD se muestra en la Tabla VI.

Tabla I. Estadística descriptiva para las variables peso del órgano y tiempo de deshidratación de cada sub-etapa.

\begin{tabular}{lcccccccc}
\hline & $\mathrm{n}$ & Media & D.E. & Var(n-1) & Mín & Máx & Asimetría & Kurtosis \\
\hline Peso (g) & 20 & 409 & 98,94 & 9788,42 & 240 & 580 & $-0,54$ & -056 \\
TD1 & 20 & 23,08 & 0,40 & 0,16 & 22,25 & 23,57 & $-0,95$ & 0,03 \\
TD2 & 20 & 62,65 & 16,21 & 262,64 & 42,58 & 94,72 & 0,60 & $-0,64$ \\
TD3 & 20 & 70,65 & 29,28 & 857,52 & 19,05 & 139,38 & 1,07 & 1,71 \\
\hline
\end{tabular}

Tabla II. Valores para la variable respuesta. A=animales adultos, $\mathrm{J}=$ animales jóvenes, $\mathrm{d}=$ =riñón derecho, i=riñón izquierdo.

\begin{tabular}{lccc}
\hline Edad / Latertalidad & TD1 & TD2 & TD3 \\
\hline A / d & 22,71 & 67,24 & 73,72 \\
J / d & 23,37 & 57,45 & 50,73 \\
A / i & 22,84 & 68,44 & 73,72 \\
J / i & 23,39 & 57,49 & 84,44 \\
\hline
\end{tabular}

Tabla III. Matriz de covarianzas común.

\begin{tabular}{lrrr}
\hline & TD1 & \multicolumn{1}{c}{ TD2 } & \multicolumn{1}{c}{ TD3 } \\
\hline TD1 & 0,07 & 1,58 & 0,68 \\
TD2 & 1,58 & 278,04 & 199,35 \\
TD3 & 0,68 & 199,35 & 828,92
\end{tabular}

Tabla IV. Análisis de la Varianza Multivariado (MANOVA). SSP = Matriz de suma de cuadrados y productos cruzados.

\begin{tabular}{lrrrr}
\hline & \multicolumn{3}{c}{ SSP } & Gl \\
\hline Factor 1: Edad & 1,789 & $-31,021$ & $-18,358$ & 1 \\
& $-31,021$ & 537,844 & 318,289 & \\
Factor 2: Lateralidad & $-18,358$ & 318,289 & 188,359 & \\
& 0,027 & 0,230 & 6,237 & 1 \\
& 0,230 & 1,935 & 52,427 & \\
Edad/Lateralidad & 6,237 & 52,427 & 1420,579 & \\
& 0,015 & 0,157 & $-4,552$ & 1 \\
& 0,157 & 1,689 & $-49,000$ & \\
Residuos: & $-4,552$ & $-49,000$ & 1421,242 & \\
& 1,150 & 25,226 & 10,909 & 16 \\
& 25,226 & 4448,674 & 3189,546 & \\
Total & 10,909 & 3189,546 & 13262,753 & \\
& 2,981 & $-5,408$ & $-5,765$ & 19 \\
& $-5,408$ & 4990,142 & 3511,262 & \\
& $-5,765$ & 3511,262 & 16292,932 & \\
\hline
\end{tabular}


RIVERA, M. C.; BONINO, F.; FIORETTI, C.; GALÁN, M.; GIGENA, S.; MOINE, R.; MOUGUELAR, H.; NATALI, J. \& QUINTEROS, R. Análisis multivariado aplicado a la etapa de deshidratación en la técnica de plastinación del riñón de caballo. Int. J. Morphol., 27(3):855-859, 2009.

Tabla V. Cuadro de análisis de la varianza (Wilks). F.V. = fuente de variación.

\begin{tabular}{lccccc}
\hline$F . V$. & Estadístico & $F$ & gl(num $)$ & gl(den) & $p$ \\
\hline Edad & 0,300 & 10,640 & 3 & 14 & 0,001 \\
Lateralidad & 0,870 & 0,730 & 3 & 14 & 0,552 \\
Edad/Lateralidad & 0,870 & 0,670 & 3 & 14 & 0,583 \\
\hline
\end{tabular}

Tabla VI. Correlacion de Spearman: coeficientes\probabilidades.

\begin{tabular}{lcccc}
\hline & Peso $(\mathrm{g})$ & TD1 & TD2 & TD3 \\
\hline Peso $(\mathrm{g})$ & 1,00 & 0,15 & 0,84 & 0,16 \\
TD1 & $-0,33$ & 1,00 & 0,97 & 0,96 \\
TD2 & 0.05 & $-0,01$ & 1,00 & 0,02 \\
TD3 & $-0,32$ & $-0,01$ & 0,56 & 1,00 \\
\hline
\end{tabular}

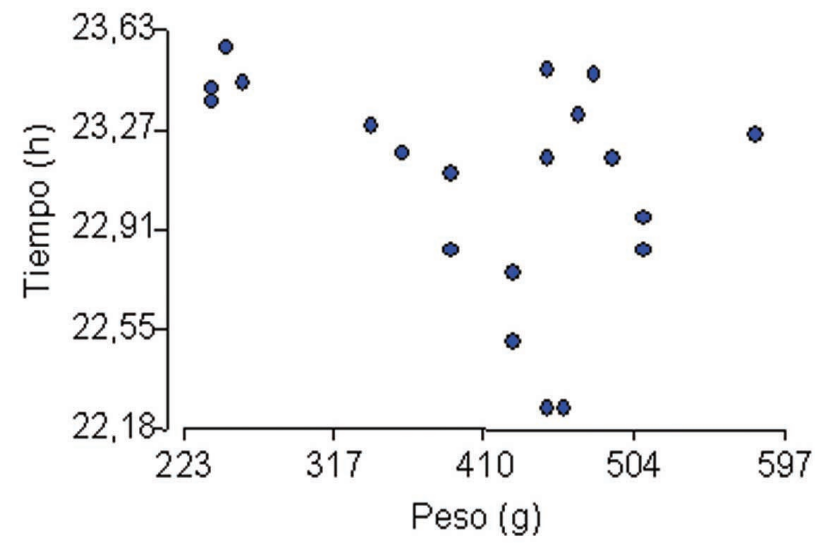

Fig. 1. diagrama de dispersión Sub-etapa 1.

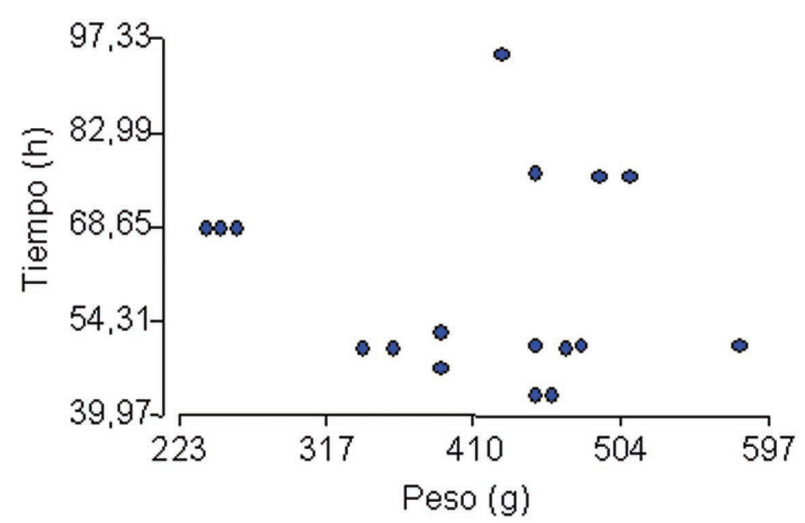

Fig. 2. diagrama de dispersión sub-etapa 2.

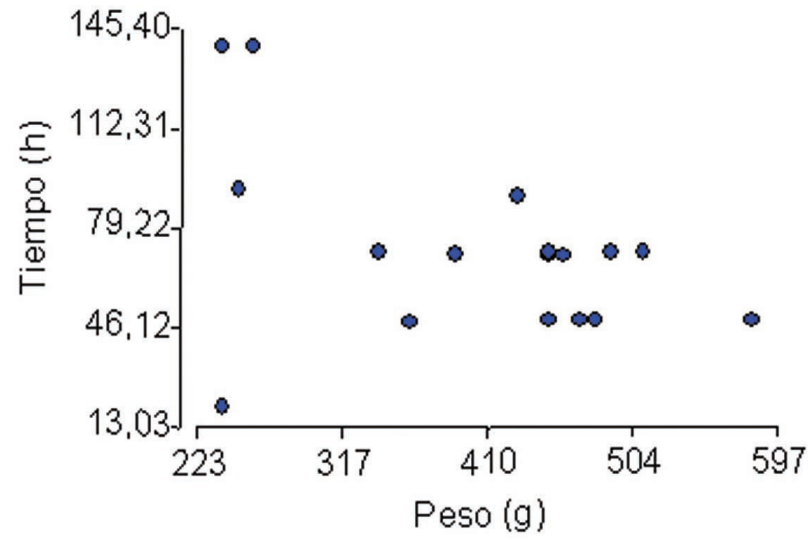

Fig. 3. diagrama de dispersión Sub-etapa 3.

\section{DISCUSIÓN}

El Análisis de la Varianza Multivariada nos permite afirmar que:

Como el p-valor para la interacción es superior a 0,05 ( $\mathrm{p}=0,583)$ no se puede rechazar la hipótesis nula de interacción. No hay evidencias estadísticamente significativas para afirmar que existe un efecto interacción entre la edad y lateralidad del órgano a un nivel del $5 \%$, por lo tanto, los factores mencionados se pueden estudiar por separado.

Como el p-valor para el factor edad es inferior a 0,05 ( $\mathrm{p}=0,001)$ se debe rechazar la hipótesis nula en el estudio de la influencia del factor edad, por consiguiente hay evidencias estadísticamente significativas para afirmar que la edad afecta el tiempo de deshidratación en cada sub-etapa a un nivel del $5 \%$.

Como el p-valor para el factor lateralidad del órgano es superior a 0,05 ( $\mathrm{p}=0,552)$ no se puede rechazar la hipótesis nula en el análisis de este factor. Se concluye entonces que no hay evidencias estadísticamente significativas como para afirmar que existe un efecto lateralidad del órgano a un nivel del $5 \%$. 
Para el factor peso del órgano se concluye que:

De la aplicación de los coeficientes de correlación de la muestra y su posterior análisis, se desprende que no existiría una asociación lineal entre el tiempo de deshidrata- ción y el peso del órgano, ya que presentan valores bajos (lejanos de \pm 1 ).

A nivel poblacional, no existe asociación lineal entre las variables ya que los p-valores de cada prueba de hipótesis superan el nivel de significación del $5 \%$.

RIVERA, M. C.; BONINO, F.; FIORETTI, C.; GALÁN, M.; GIGENA, S.; MOINE, R.; MOUGUELAR, H.; NATALI, J. \& QUINTEROS, R. Multivaried analysis at the stage of dehydration in the in the plastination technique in horse's kidney. Int. J. Morphol., 27(3):855-859, 2009.

SUMMARY: Plastination is the most modern technique for the preservation of anatomical pieces and specimens. This technique consists of four stages: fixation, dehydration, forced impregnation, and cured. This research aims at improving the efficiency of production plastinated organs through the standard technique known as S10. Kidneys of criole mestizo equines from Frigorifico Aimar S.A placed in the rural area of Rio IV were used. After binding the organ with formalin, it was dehydrated. The dehydration was carried out in three substages working at the same temperature, and a solvent composed of solution of acetone of different concentrations was used. The measurements were analysed with acetometer, keeping register of the time consumption to reach dehydration. The resulting data was statistically analysed using Infostat program. Results showed that the time consumed to reach dehydration was influenced by the horses age but not by the kidney's weight, there were no significant differences between right and left kidneys neither.

KEY WORDS: Plastination; Acetona; Dehydration; Kidney; Horse.

\section{REFERENCIAS BIBLIOGRÁFICAS}

Ballenguer, J. J. Some effects of formaldehyde on the upper respiratry tract. Laringoscop., 94:1411-3, 1984.

von Hagens, G.; Tiedemann, K. \& Kriz, W. The current potential of plastination. Anat. Embryol. (Berl), 175:411$21,1987$.

Olsen, J. H.; Jersen, S.; Hink, M.; Faurbo, K.; Breum, N. O. \& Jensen, O. M. Occupational Formaldehyde exposure and increased nasal cancer risk in man. Int. J. Cancer, 34:639-44, 1984.
Dirección para correspondencia:

Natali, J.

Anatomía Animal

Depto. de Anatomía Animal. Facultad de Agronomía y Veterinaria. Universidad Nacional de Río Cuarto ARGENTINA

Email: Jnatali@ayv.unrc.edu.ar

Recibido : 09-01-2009

Aceptado: 11-08-2009 
